\title{
Integrating Phylodynamic Techniques for Next-generation HIV Surveillance in Florida
}

\author{
Shannan N. Rich ${ }^{2}$, Carla Mavian ${ }^{1}$, Veronica Richards ${ }^{2}$, Robert Cook ${ }^{2}$, Mattia Prosperi ${ }^{2}$, Marco \\ Salemi ${ }^{1}$ \\ ${ }^{1}$ Emerging Pathogens Institute, Department of Pathology, Immunology, and Laboratory Medicine, University of Florida, Gainesville, Florida, United \\ States, ${ }^{2}$ Department of Epidemiology, College of Public Health and Health Professions and College of Medicine, University of Florida, Gainesville, \\ Florida, United States
}

\section{Objective}

We aim to 1) develop and implement a novel theoretical and technical framework able to dynamically model HIV transmission clusters in near-real time; 2) validate the model with real data; and 3) host focus groups with governmental stakeholders to identify optimal strategies for precision public health interventions.

\section{Introduction}

Reducing HIV incidence requires a 'precision public health' approach encompassing prevention campaigns, targeted interventions, and 'next- generation' surveillance through multimodal instruments, including sequencing. Molecular epidemiology methods (phylogenetics and phylodynamics) have recently gained traction for use in identifying and tracking epidemic transmission clusters, as well as reconstructing the demographic history of viral pathogen populations. However, such methods are not equipped to identify both transmission clusters and their corresponding dynamics in real time, and transmission clusters are assumed to be unrealistically static over the course of the epidemic. We will focus on the ongoing HIV epidemic in Florida, which has one of the highest HIV incidence rates in the United States. Although key HIV transmission risk groups have been identified in Florida through classical epidemiology surveillance methods, there remains a critical need for detection and tracking of expanding transmission clusters in near-real time.

\section{Methods}

We propose to develop and test a new phylodynamic method, HIV Dynamic Identification of Transmission Epicenters (HIV DYNAMITE), that will support existing HIV surveillance efforts. In collaboration with the Florida Department of Health (FDOH), we will leverage an existing dataset, which contains over 44,300 sequences, and apply HIV-DYNAMITE to identify transmission clusters and infer growth trends of these clusters within epidemics. HIV-DYNAMITE will also be used to identify and predict infection trends and virus spread by conferring with demographic data. The system will be validated using newly obtained longitudinal data. Focus group discussions with the FDOH, the Centers for Disease Control and Prevention (CDC), and other stakeholders will be conducted to confer how to employ HIV-DYNAMITE into statewide informatics systems and to design future intervention strategies.

\section{Results}

These methods are still under development.

\section{Conclusions}

In conclusion, this study aims to both complement and enhance existing efforts, such as the CDC's HIV-TRACE, which is currently based on sequence data alone and lacks dynamic or geographic spread components. This approach has the potential to be incorporated into other settings within the US with comparable statewide surveillance and virus sequencing coverage through national reference centers. 


\section{Acknowledgement}

This project was supported by the Florida Department of Health and the National Institute of Allergy and Infectious Diseases. 